\title{
À quels choix les jeunes sont-ils confrontés lors de leur première inscription dans l'enseignement tertiaire?
}

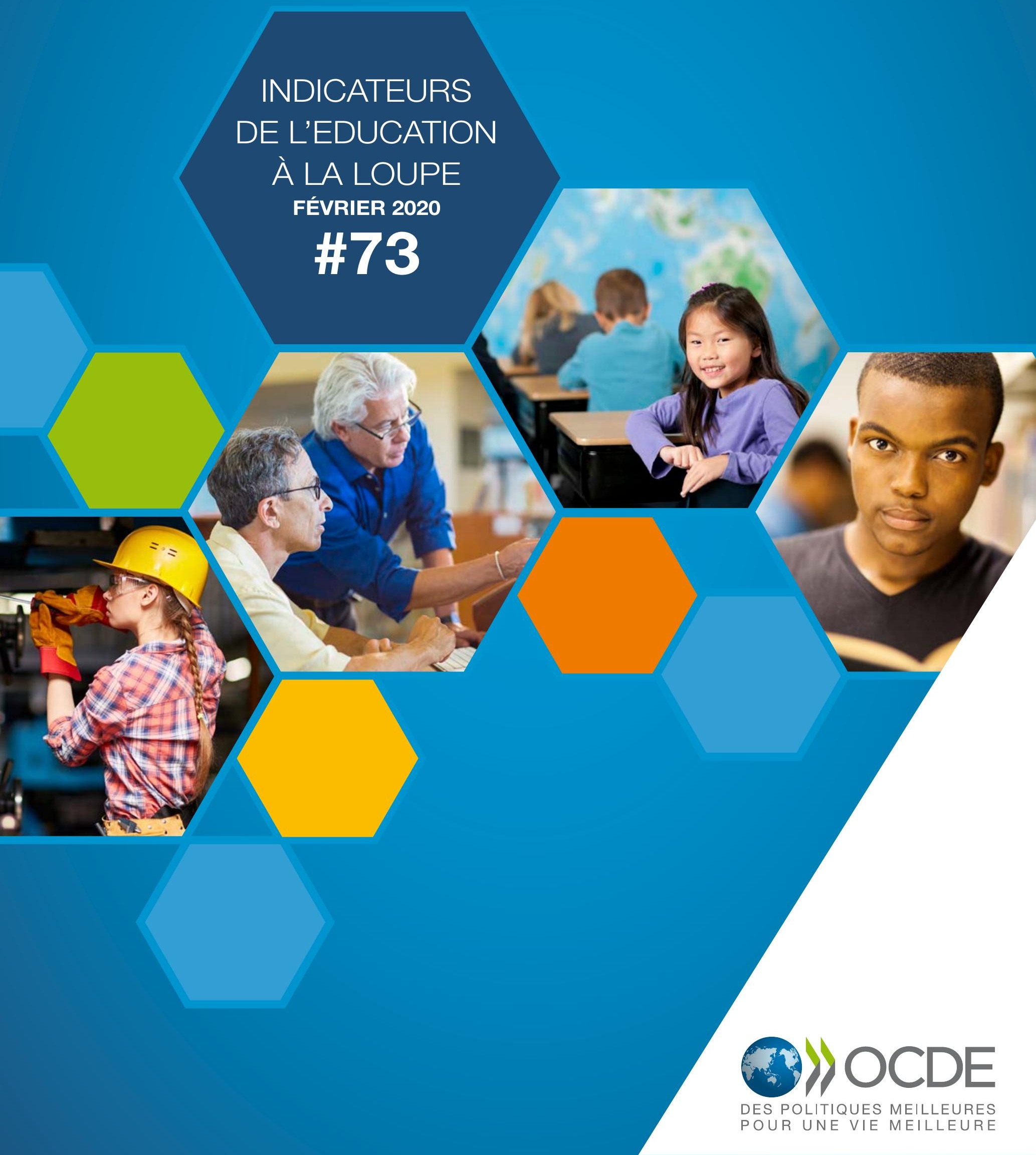


- Le domaine d'études choisi par les nouveaux inscrits dans l'enseignement tertiaire-aura certes une incidence sur leurs perspectives d'emploi et de revenus, mais pas autant que certains pourraient le croire. Dans les pays de l'OCDE, le taux d'emploi moyen des diplômés de ce niveau d'enseignement va ainsi de $90 \%$ parmi ceux qui ont étudié les technologies de l'information et de la communication, à 83 \% pour ceux qui ont choisi une formation en lettres et arts, sciences sociales, journalisme et information.

- Le niveau des frais de scolarité à charge des étudiants et des aides financières dont ils bénéficient dépend de l'endroit où ils étudient. Les frais de scolarité annuels moyens (ou les plus courants) sont ainsi nuls dans les établissements publics de plusieurs pays de l'OCDE, mais peuvent s'élever jusqu'à 29478 USD $^{1}$ dans les établissements privés indépendants aux États-Unis. En moyenne, le rendement financier de l'obtention d'un diplôme de l'enseignement tertiaire excède largement son coût privé.

- Les critères utilisés pour évaluer l'aptitude des candidats à suivre un cursus donné varient considérablement. Dans la plupart des pays de l'OCDE, ils devront réussir un examen d'entrée, mais au-delà de ce critère, aucune uniformité particulière ne se dégage des modalités de sélection. Les entretiens, les lettres de motivation et les résultats du secondaire sont autant de critères utilisés à des degrés divers dans les différents pays.

De nombreux facteurs influeront sur le choix des diplômés du deuxième cycle du secondaire d'entamer des études supérieures, notamment leur milieu économique, social et culturel, mais aussi leurs intérêts, motivations et compétences à titre personnel. Les candidats utiliseront différents raisonnements pour faire leur choix et accorderont plus ou moins d'importance à différentes variables, tout en disposant toujours d'informations imparfaites sur les conséquences des décisions qu'ils prennent. Ces nouveaux inscrits ne peuvent en effet pas évaluer avec certitude leurs chances de réussir tel ou tel cursus, ou encore si les compétences et connaissances qu'ils auront acquises à l'obtention de leur diplôme seront valorisées sur le marché du travail. Toutefois, malgré ce lot d'incertitudes, la poursuite d'études supérieures reste un investissement sûr.

Sur le plan des facteurs économiques, les candidats à un premier cursus dans l'enseignement tertiaire doivent faire des choix interdépendants concernant leur domaine d'études, les frais qu'ils devront assumer et les critères de sélection qu'ils sont disposés à satisfaire. Le domaine d'études qu'ils retiendront aura en effet une incidence sur leurs perspectives d'emploi et de revenus. Les dettes éventuellement contractées pendant leurs études pourront par ailleurs limiter leurs choix futurs en termes de carrière et de lieu de vie. Enfin, loin d'être totalement libres du choix de l'établissement ou du domaine d'études qui les intéressent le plus, ils devront faire preuve de leurs compétences par le biais d'une combinaison d'examens, de résultats du secondaire, d'entretiens et de lettres de motivations.

\section{Les domaines d'études ont une incidence sur les revenus et les taux d'emplois futurs des étudiants}

Dans de nombreux cas, les nouveaux inscrits dans l'enseignement tertiaire passent d'un cursus secondaire généraliste à un domaine d'études bien plus spécialisé. Dans l'enseignement tertiaire, les grands domaines d'études les plus souvent associés aux taux d'emploi les plus élevés sont l'ingénierie, les industries de transformation et la construction, et les technologies de l'information et de la communication (TIC). En moyenne, dans les pays de l'OCDE, les diplômés dans le domaine des TIC ont ainsi un taux d'emploi de $90 \%$, contre $83 \%$ pour ceux du domaine des lettres et arts, sciences sociales, journalisme et information, soit un écart de 7 points de pourcentage, certes significatif, mais peut-être pas aussi marqué que beaucoup pourraient le croire (graphique 1). La quasi-totalité des pays de l'OCDE présentent un écart similaire entre ces deux grands domaines d'études.

Le choix de domaine d'études que les diplômés de l'enseignement tertiaire auront fait peut en outre influer sur leurs revenus futurs. Ceux qui ont opté pour celui de l'éducation, ou des lettres et arts, sciences sociales, journalisme et information, peuvent ainsi escompter des revenus inférieurs à ceux qui auront choisi l'ingénierie, les industries de transformation et la construction, ou les TIC. À titre d'exemple, en Allemagne, les diplômés de l'enseignement tertiaire ayant suivi un cursus en éducation gagnent 30 \% de plus que les 
Graphique 1 / Taux d'emploi des adultes diplômés de l'enseignement tertiaire, selon le domaine d'études (2018)

Pourcentage d'actifs occupés âgés de 25 à 64 ans dans l'ensemble de la population âgée de 25 à 64 ans

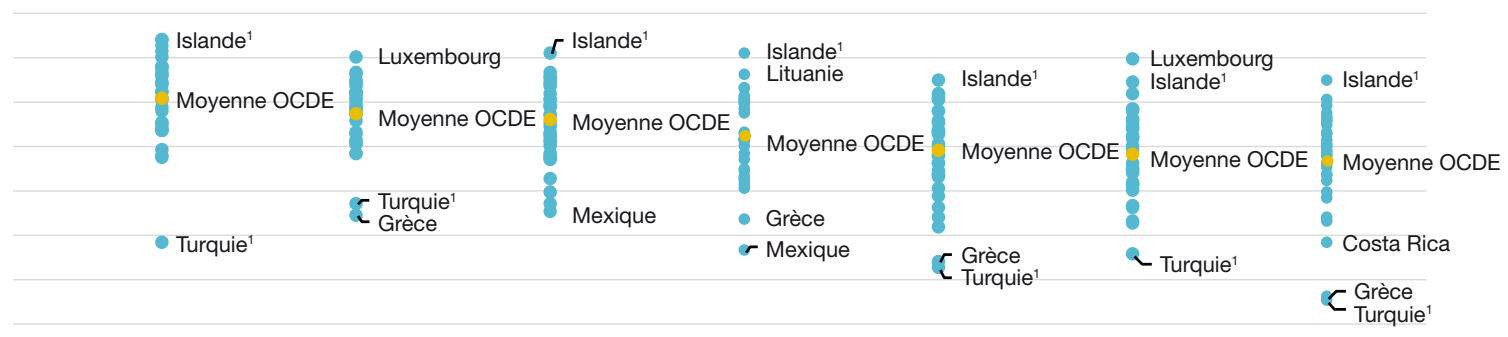

\begin{tabular}{|c|c|c|c|c|c|c|}
\hline $\begin{array}{l}\text { Technologies de } \\
\text { l'information et de la } \\
\text { communication }\end{array}$ & $\begin{array}{l}\text { Ingénierie, industries } \\
\text { de transformation } \\
\text { et construction }\end{array}$ & $\begin{array}{c}\text { Santé et protection } \\
\text { sociale }\end{array}$ & $\begin{array}{c}\text { Commerce, } \\
\text { administration et } \\
\text { droit }\end{array}$ & Éducation & $\begin{array}{l}\text { Sciences naturelles, } \\
\text { mathématiques et } \\
\text { statistiques }\end{array}$ & $\begin{array}{l}\text { Lettres et arts, } \\
\text { sciences sociales, } \\
\text { journalisme et } \\
\text { information }\end{array}$ \\
\hline
\end{tabular}

1. L'année de référence n'est pas 2018. Consulter Regards sur l'éducation 2019 pour de plus amples informations.

Les domaines d'études sont classés par ordre décroissant des taux d'emploi pour la moyenne OCDE.

Source : OCDE (2019 [1] ), Regards sur l'éducation 2019 : Les indicateurs de l'OCDE, https://doi.org/10.1787/6bcf6dc9-fr.

adultes qui n'ont qu'un diplôme du deuxième cycle du secondaire, contre 116 \% de plus pour les diplômés de l'enseignement tertiaire qui ont opté pour l'ingénierie, les industries de transformation et la construction.

De nombreux pays limitent le nombre de places dans certains domaines d'études, voire tous. Parmi les 36 pays membres ou partenaires de l'OCDE disposant de données, 25 appliquent ainsi ce type de limitations dans les établissements publics. Cette pratique est en revanche moins courante dans les établissements privés indépendants. Ces restrictions peuvent servir à adapter le nombre de diplômés aux besoins du marché du travail, mais aussi refléter les limites de capacité d'accueil des établissements.

De nombreux aspirants aux études supérieures choisissent ainsi leur domaine d'études dans des systèmes où le nombre de places est encadré par des politiques gouvernementales ou des décisions institutionnelles. S'il est évident que les domaines d'études débouchant sur des professions mieux rémunérées (médecine, droit, commerce et administration) seront plus prisés, il est en revanche moins sûr que le nombre de places en sciences naturelles ou en ingénierie sera à la hauteur de cette demande. Les candidats doivent par ailleurs non seulement prendre en compte les chances qu'ils ont d'obtenir une place dans le domaine d'études visé, mais aussi d'y réussir. Car, au bout du compte, ils n'en récolteront les fruits en termes de taux d'emploi et de revenus qu'une fois leur diplôme décroché.

\section{Les frais de scolarité varient considérablement entre les pays et au sein de ceux-ci}

Les coûts associés à une formation tertiaire sont un facteur important dans le choix des diplômés du deuxième cycle du secondaire de poursuivre ou non leurs études. Ces coûts comprennent les frais de scolarité, ainsi que d'autres coûts directs et indirects.

Les frais de scolarité demandés par les établissements varient considérablement entre les pays (graphique 2). Dans environ un tiers, les établissements publics n'en facturent aucun aux ressortissants nationaux en licence ou formation de niveau équivalent. Dans un autre tiers, les frais de scolarité à charge de ces étudiants sont faibles ou modérés (inférieurs à 2600 USD par an). Et enfin, dans le tiers restant, ils sont élevés, allant de 3000 à plus de 9000 USD par an. 
Graphique 2 / Frais de scolarité annuels minimums, maximums et moyens (ou les plus courants) demandés par les établissements publics aux ressortissants nationaux en licence ou formation de niveau équivalent $(2017 / 18)$

Frais de scolarité annuels demandés aux ressortissants nationaux scolarisés à temps plein, en équivalents USD convertis sur la base des PPA pour le PIB

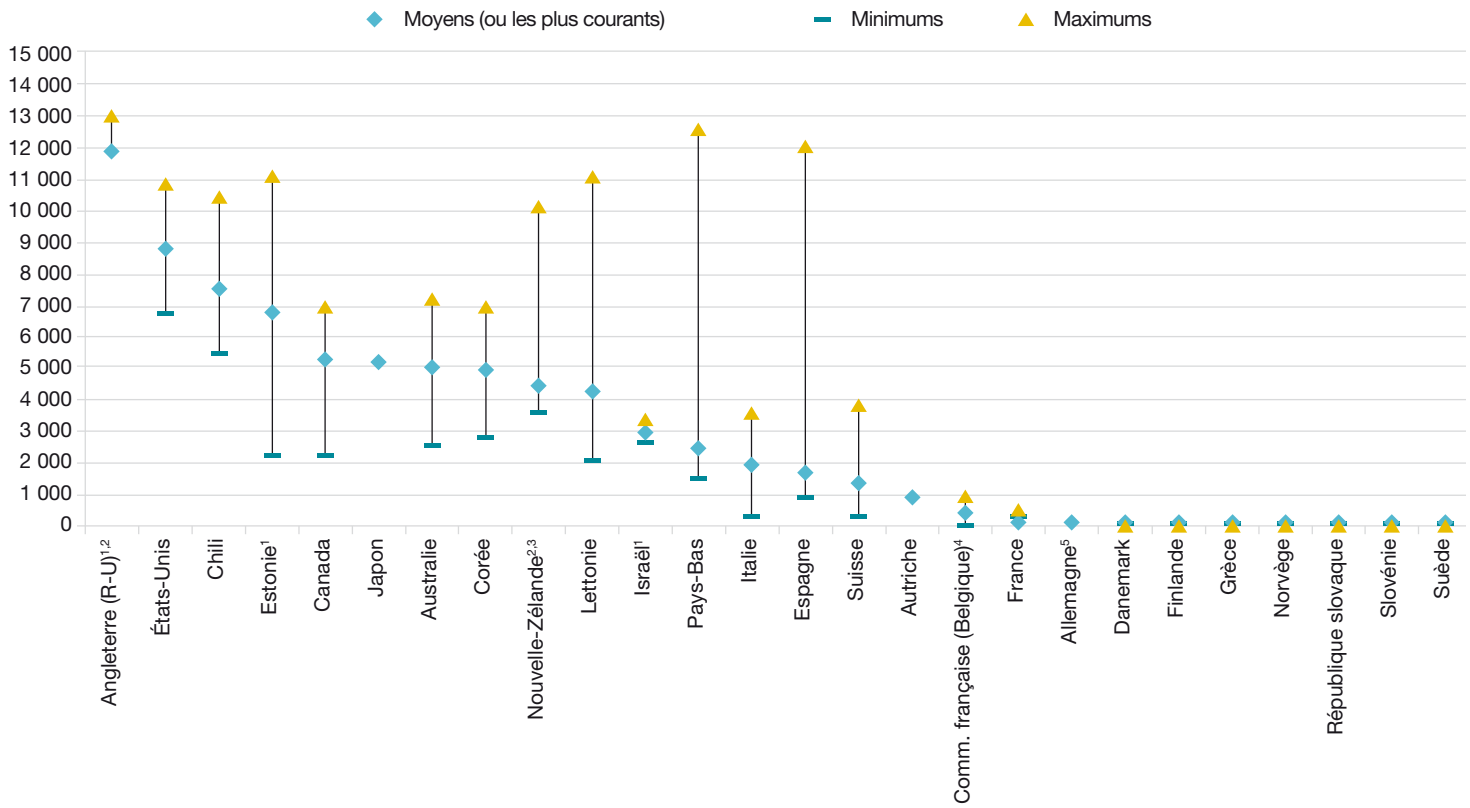

Remarque : L'année de référence peut différer en fonction des pays et économies. Consulter l'annexe 3 de Regards sur l'éducation 2019 pour de plus amples informations.

1. Les données sont indiquées pour les établissements privés subventionnés par l'État, et non pour les établissements publics.

2. Les formations tertiaires de cycle court sont incluses dans la catégorie des formations de licence ou de niveau équivalent.

3. Les estimations incluent uniquement les universités et excluent certaines formations. Consulter Regards sur l'éducation 2019 pour de plus amples informations.

4. Les établissements privés subventionnés par l'État sont inclus dans la catégorie des établissements publics.

5. Les frais de scolarité sont indiqués pour l'enseignement tertiaire dans son ensemble, et non pour la licence ou niveau équivalent. II s'agit des frais de scolarité demandés aux ressortissants nationaux et étrangers. Seuls sont inclus les programmes académiques.

Les pays et économies sont classés par ordre décroissant des frais de scolarité annuels moyens (ou les plus courants).

Source : OCDE $\left(2019_{[1]}\right)$, Regards sur l'éducation 2019 : Les indicateurs de l'OCDE, https://doi.org/10.1787/6bcf6dc9-fr.

Toutefois, même dans les pays où les frais de scolarité sont élevés, les avantages financiers de l'obtention d'un diplôme de l'enseignement tertiaire peuvent largement en excéder les coûts. De fait, dans tous les pays de l'OCDE, le rendement financier net de l'obtention d'un diplôme de ce niveau d'enseignement est fortement positif par rapport à celui de l'obtention d'un diplôme du deuxième cycle du secondaire, s'élevant en moyenne à 295900 USD pour les hommes et à 227600 USD pour les femmes. Ce rendement varie toutefois considérablement selon les pays, les domaines d'études et les établissements d'enseignement. Or, cette variation pourra en dissuader certains de poursuivre des études supérieures, notamment en cas d'accès limité aux aides financières.

Dans certains pays de l'OCDE, les étudiants doivent décider s'ils souhaitent s'inscrire dans un établissement public (dont les frais de scolarité sont généralement plus faibles) ou dans un établissement privé indépendant (où les frais de scolarité tendent à être plus élevés). L'écart peut être assez important : aux États-Unis, les frais de scolarité annuels à acquitter pour une licence ou une formation de niveau équivalent sont par exemple de 8804 USD dans les établissements publics, contre 29748 USD dans les établissements privés indépendants. Les étudiants doivent donc décider si les avantages (financiers ou autres) de suivre un cursus dans un établissement privé indépendant l'emportent sur ces coûts supplémentaires.

Cependant, tous les étudiants des pays de l'OCDE ne sont pas confrontés à un tel choix. La taille du secteur privé indépendant varie en effet considérablement d'un pays à l'autre. Dans des pays comme les États-Unis, la Lettonie et le Portugal, un cinquième à un tiers des étudiants (en licence ou formation de niveau équivalent) 
Graphique 3 / Finalité des examens nationaux/centraux et utilisation de leurs résultats comme critère d'admission dans les établissements d'enseignement tertiaire (2017)

Par examens nationaux/centraux, on entend ceux administrés aux élèves au terme du deuxième cycle du secondaire

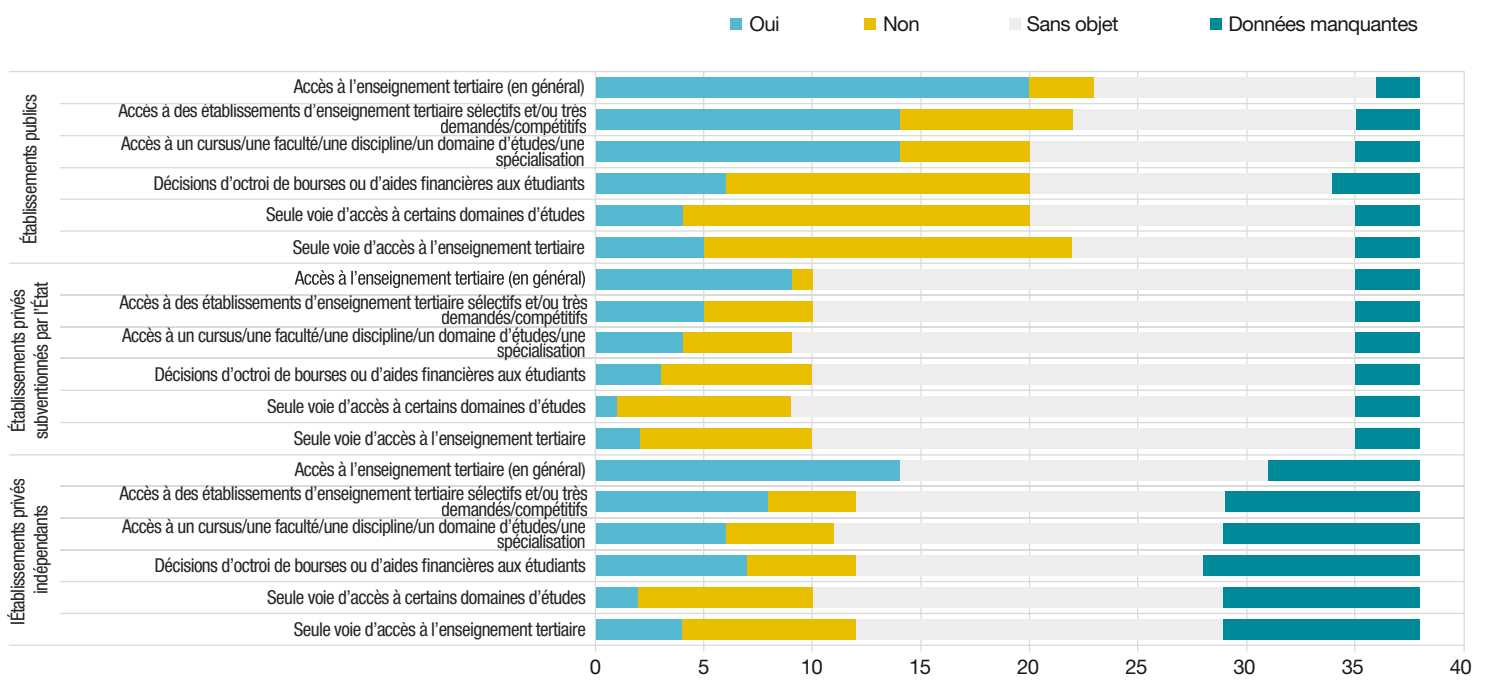

Source : OCDE (2019 $\left.{ }_{[1]}\right)$, Regards sur l'éducation 2019 : Les indicateurs de l'OCDE, https://doi.org/10.1787/6bcf6dc9-fr.

sont inscrits dans des établissements privés indépendants, une proportion qui dépasse même $60 \%$ au Chili, en Corée et au Japon. À l'inverse, dans plusieurs pays ou économies de l'OCDE - Angleterre (Royaume-Uni), Canada, Finlande, Grèce et Suède -, aucun établissement privé indépendant ne scolarise des étudiants en licence ou formation de niveau équivalent

Les étudiants peuvent également souhaiter étudier à l'étranger. Cependant, les étudiants étrangers doivent souvent s'acquitter de frais de scolarité plus élevés que les ressortissants nationaux pour le même cursus dans le même établissement. Les étudiants qui partent suivre un cursus à l'étranger risquent en outre de ne pas bénéficier des mêmes aides financières que s'ils étaient restés dans leur pays. Du fait de ces deux aspects, moins d'étudiants sont susceptibles de faire ce choix que si ces obstacles étaient levés. De nombreux étudiants restent toutefois clairement motivés pour partir étudier à l'étranger, que ce soit parce que des aides financières leur sont proposées ou qu'ils disposent d'un plus grand choix de cursus, ou encore que le nombre de places à pourvoir soit moins limité.

\section{Les systèmes d'admission varient fortement entre les pays}

Le processus d'admission dans l'enseignement tertiaire varie considérablement entre les pays de l'OCDE. Les systèmes d'éducation diffèrent tous par le degré de centralisation de ce processus, le nombre de préférences que les candidats peuvent indiquer, le nombre de propositions qu'ils peuvent recevoir et les critères utilisés pour la sélection des étudiants.

Dans certains pays, les candidatures se font directement auprès des établissements, tandis que dans d'autres, elles passent par un système centralisé (ou une combinaison de ces deux modalités). Parmi les pays membres ou partenaires de l'OCDE disposant de données, 16 laissent les établissements publics gérer directement ces candidatures, 11 autres disposent d'un système mixte combinant candidatures directes et centralisées, et les 10 restants ont un système centralisé.

Dans de nombreux pays dotés de systèmes d'admission centralisés, le nombre de préférences que les candidats peuvent indiquer est limité, allant de 2 au Brésil à 24 en France et en Turquie. Les seuls pays dotés de systèmes centralisés ou mixtes ne limitant pas le nombre de préférences sont la Grèce, l'Italie et la Nouvelle-Zélande. 
Le nombre de propositions faites aux candidats peut par ailleurs également être limité. Dans les systèmes d'admission centralisés, il est courant de n'autoriser qu'une seule proposition par candidat. La Fédération de Russie et les Pays-Bas autorisent quant à eux trois propositions par candidat, tandis que l'Australie, le Canada, la Corée et l'Italie ne fixent aucune limite en la matière.

Les candidats à un cursus dans un établissement privé indépendant sont bien moins susceptibles de se trouver confrontés à de telles restrictions. Dans 10 pays seulement, le système d'admission dans ce type d'établissement est centralisé, tandis que dans 18 autres, les candidatures directes sont autorisées. Lorsqu'il existe une forme de centralisation, quel qu'en soit le degré, les limitations des préférences et propositions sont similaires à celles appliquées aux établissements publics.

Les candidats à un cursus de l'enseignement tertiaire peuvent s'attendre à devoir faire preuve de leurs aptitudes de diverses manières. Cela signifie que le choix de leur cursus et/ou de leur établissement est susceptible d'être influencé par les critères et le processus d'admission. Les examens nationaux ou centraux sont les critères les plus couramment appliqués pour l'évaluation des candidats, mais leur utilisation n'est pas généralisée (graphique 3). Parmi les 26 pays membres ou partenaires de l'OCDE disposant de données sur les systèmes d'admission dans les établissements publics, 21 ont recours aux examens nationaux ou centraux. Ces examens évaluent les compétences des candidats à un moment donné, généralement à la fin ou vers la fin du deuxième cycle du secondaire,

Les candidats peuvent aussi avoir à présenter leurs notes moyennes du secondaire, ce qui signifie qu'ils sont évalués sur plusieurs années. Ce critère est utilisé dans les établissements publics de 19 pays membres ou partenaires de l'OCDE parmi les 29 disposant de données.

Les candidats peuvent également être invités à passer un entretien. Les établissements publics de 20 pays membres ou partenaires de l'OCDE utilisent ce critère, tandis que 7 n'y ont pas recours. Certains pays utilisent d'autres critères tels que les lettres de motivation, l'expérience professionnelle ou encore les revenus familiaux des candidats, mais dans une bien moindre mesure que les examens, les notes moyennes du secondaire et les entretiens. L'importance accordée à ces différents critères varie selon les pays, mais globalement, les notes moyennes comptent plus que les entretiens pour l'admission dans les établissements publics.

Il est quelque peu surprenant que de telles différences de systèmes d'admission existent entre les pays à l'heure où la mobilité internationale des étudiants ne cesse d'augmenter. Au cours des 20 dernières années, le nombre d'étudiants en mobilité internationale dans l'enseignement tertiaire a connu un essor sans précédent dans le monde, passant de 2 millions en 1998 à 5.3 millions en 2017, avec un taux de croissance annuel moyen de $5 \%$ dans les pays de l'OCDE. Les processus et critères d'admission restent toutefois très hétérogènes et il n'est pas certain que les candidats rompus aux exigences d'un système soient capables de maîtriser celles d'un autre. 


\section{Pour conclure}

Les candidats à un premier cursus dans l'enseignement tertiaire doivent faire des choix interdépendants concernant leur domaine d'études, les frais qu'ils devront assumer et les critères de sélection qu'ils sont disposés à satisfaire. Le choix de leur cursus et de leur établissement sera influencé par les différents critères de coûts et d'admission, et aura une incidence à long terme sur leurs perspectives d'emploi et de revenus.

\section{RÉFÉRENCES :}

[1] OCDE (2019), Regards sur l'éducation 2019: Les indicateurs de l'OCDE, Éditions OCDE, Paris, https:// doi.org/10.1787/6bcf6dc9-fr.

$\begin{array}{ll}\text { VOIR } & \text { www.oecd.org/education/education-at-a-glance-19991487.htm } \\ & \begin{array}{l}\text { Indicateurs de l'éducation à la loupe (numéros précédents) } \\ \text { PISA à la loupe } \\ \text { L'enseignement à la loupe }\end{array}\end{array}$

PROCHAIN NUMÉRO Comment les taux de scolarisation des femmes dans l'enseignement supérieur et leurs choix de domaines d'études ont-ils évolué au fil du temps?

\section{CONTACTER:}

Bruce Golding (bruce.golding@oecd.org)

Crédit photo : @ Christopher Futcher / iStock ; @ Marc Romanelli / Gettyimages ; @ michaeljung / Shutterstock ; @ Pressmaster / Shutterstock. Ce document est publié sous la responsabilité du Secrétaire général de l'OCDE. Les opinions qui y sont exprimées et les arguments qui y sont employés ne reflètent pas nécessairement les vues officielles des pays membres de l'OCDE.

Ce document, ainsi que les données et cartes qu'il peut comprendre, sont sans préjudice du statut de tout territoire, de la souveraineté s'exerçant sur ce dernier, du tracé des frontières et limites internationales, et du nom de tout territoire, ville ou région.

Les données statistiques concernant Israël sont fournies par et sous la responsabilité des autorités israéliennes compétentes. L'utilisation de ces données par l'OCDE est sans préjudice du statut des hauteurs du Golan, de Jérusalem-Est et des colonies de peuplement israéliennes en Cisjordanie aux termes du droit international. 